Annuaire suisse de politique de développement

\title{
Eclairage - Les transferts de fonds informels des Afghans : une dispersion stratégique
}

\section{Alessandro Monsutti}

\section{(2) OpenEdition}

1 Journals

Édition électronique

URL : http://journals.openedition.org/aspd/520

DOI : 10.4000/aspd.520

ISSN : 1663-9669

Éditeur

Institut de hautes études internationales et du développement

\section{Édition imprimée}

Date de publication : 1 décembre 2008

Pagination : 155-159

ISBN : 978-2-940415-07-6

ISSN : 1660-5934

\section{Référence électronique}

Alessandro Monsutti, «Eclairage - Les transferts de fonds informels des Afghans : une dispersion stratégique », Annuaire suisse de politique de développement [En ligne], 27-2 | 2008, mis en ligne le 18 mars 2010, consulté le 10 décembre 2020. URL : http://journals.openedition.org/aspd/520 ; DOI : https://doi.org/10.4000/aspd.520 


\title{
Eclairage \\ Les transferts de fonds informels des Afghans: une dispersion stratégique
}

\author{
Alessandro Monsutti*
}

\section{L}

e conflit qu'a connu I'Afghanistan après le coup d'Etat communiste de 1978 et I'intervention soviétique de 1979 a provoqué l'un des plus massifs mouvements de population de la fin du XXe siècle. Au début des années 1990, le Haut-Commissariat des Nations unies pour les réfugiés (HCR) comptabilisait plus de 6 millions de réfugiés afghans, en grande majorité répartis entre le Pakistan et I'Iran'. IIs constituaient alors la plus importante population déplacée de toute la planète et près de la moitié des personnes tombant sous le mandat de l'agence onusienne. Toutefois, cet exode n'a pas toujours eu la dimension traumatique que tendent à lui prêter les organisations humanitaires et les médias. Une perspective historique de longue durée conduit d'ailleurs à en relativiser le caractère exceptionnel ${ }^{2}$. Nomades qui mènent au gré des saisons leurs troupeaux à la recherche des meilleurs pâturages, mais qui tirent profit de leurs déplacements pour commercer avec les sédentaires, populations montagnardes qui se rendent en ville ou dans les basses terres pour trouver un emploi saisonnier, pèlerins, fuyards ou conquérants, les Afghans ont une longue expérience de la migration sous toutes ses formes.
Aujourd'hui comme par le passé, les Afghans - à l'instar d'autres groupes de migrants et de réfugiés - envoient d'importantes sommes d'argent à leurs proches restés au pays. II est intéressant de se pencher sur ces transferts de fonds pour plusieurs raisons. Tout d'abord, ils permettent de comprendre que la fuite devant la violence n'est pas toujours incompatible avec une véritable stratégie migratoire. Ensuite, ils ont des conséquences sociales et économiques importantes pour les régions dont proviennent les réfugiés. Finalement, ils stimulent et orientent les déplacements futurs, car les réfugiés fournissent à leurs proches restés dans la région d'origine des informations sur les possibilités qui existent en différents endroits. Qu'ils soient effectués par des migrants ou par des réfugiés, les transferts de fonds révèlent ainsi l'existence de réseaux sociaux qui relient des lieux éloignés. Dans le contexte afghan, la dispersion n'est pas qu'un effet douloureux de la guerre et de la pauvreté, elle résulte aussi d'un choix planifié par lequel les membres des groupes familiaux cherchent à diversifier leurs moyens de subsistance tout en diminuant les risques liés à l'insécurité dans laquelle ils évoluent autant dans leur pays d'origine (combats persistants) que dans les

* Chargé de cours à l'Institut de hautes études internationales et du développement, Genève.

1 II fallait également ajouter environ un million et demi de personnes déplacées de l'intérieur. R. Colville, "Afghan Refugees: Is International Support Draining Away after Two Decades in Exile?», Refuge, vol. 17, n² 4, 1998, pp. 6-11.

2 M. J. Hanifi, "Anthropology and the Representations of Recent Migrations from Afghanistan», in E.M. Gozdziak and D.J. Shandy (eds.), Rethinking Refuge and Displacement: Selected Papers on Refugees and Immigrants, vol. 8, Arlington, American Anthropological Association, 2000, pp. 291-321. 
pays de destination (contrôles policiers, vagues d'expulsion).

Un regard renouvelé sur les flux migratoires permet de comprendre qu'ils ne sont bien souvent ni définitifs ni temporaires; il est plus pertinent de parler de mouvements récurrents et multidirectionnels. Cet aspect est illustré de façon spectaculaire par le cas qui nous occupe. D'un côté, rares sont les réfugiés afghans qui ne sont jamais retournés dans leur pays après leur premier départ; de l'autre, peu de groupes familiaux n'ont pas au moins un de leurs membres à l'étranger. La migration et l'exil ne sont donc pas suivis d'une intégration dans le pays d'accueil ou d'un retour définitif dans le pays d'origine, les mouvements sont continus et une véritable communauté transnationale finit par se constituer. Les Afghans ont mis en place un "territoire circulatoire», pour reprendre une expression du sociologue Alain Tarrius ${ }^{3}$.

Le cas des Hazaras est à ce titre exemplaire. Originaires du centre montagneux et peu fertile de l'Afghanistan, ils sont en grande partie de religion chiite et considérés avec suspicion et mépris par la majorité sunnite de la population. Poussés par la pauvreté et la marginalité politique, ils ont été nombreux à quitter leur région d'origine par le passé à la recherche d'emplois dans les villes afghanes ou dans les pays limitrophes que sont I'Iran et le Pakistan, mais aussi les Emirats arabes unis, le Koweït ou l'Arabie saoudite. Ces mouvements anciens ont pris une ampleur sans précédent au cours de la guerre. Les Hazaras - à l'instar d'autres segments de la population afghane - ont tissé de très efficaces réseaux migratoires articulés sur la dispersion des membres des groupes de parenté entre I'Iran, le Pakistan et I'Afghanistan. De façon croissante, certaines personnes se sont établies en Europe, en Amérique du Nord, en Australie et en Nouvelle-Zélande. Ce vaste ensemble forme un véritable système transnational et il est difficile de comprendre les stratégies développées dans un endroit sans se référer à celles qui prévalent ailleurs. Chaque emplacement présente des avantages et des inconvénients. En Afghanistan, les Hazaras ont connu un processus d'émancipation politique, mais la situation générale du pays reste sombre, autant au niveau de la sécurité que des activités économiques. Au Pakistan, les Hazaras se sont rendus en grande majorité dans la capitale du Baloutchistan, Quetta. Ils y ont bénéficié d'un milieu d'accueil en raison de la présence d'une communauté hazara établie à la fin du XIX siècle, lorsque la ville était rattachée aux Indes britanniques. Les migrants et réfugiés ont pu y évoluer assez librement, mais peu de débouchés économiques étaient accessibles. En Iran, il est en revanche assez facile de trouver un petit emploi dans le domaine de la construction, dans une minoterie ou encore comme travailleur agricole. Par contre, la pression croissante des autorités de la République islamique et les vagues périodiques d'expulsion ne permettent guère de s'y installer de façon durable en famille.

Lorsqu'un migrant hazara qui s'est rendu en Iran veut envoyer ses quelques économies à sa famille restée en Afghanistan, il ne peut faire appel au système bancaire formel. En premier lieu, il ne possède souvent pas de papiers d'identité officiels. Ensuite, si le réseau bancaire en Afghanistan s'étend progressivement depuis la chute des talibans en 2001, il était inexistant au Hazaradjat dans les années 1980 et 1990. Le migrant va dès lors confier son argent à un homme d'affaires spécialisé dans les transferts de fonds informels, nommé localement hawâladâr, de hawâla, "transfert (d'argent)", mais aussi "lettre de crédit» ou "chèque». Dans la mesure du possible, le migrant s'adresse à un hawâladâr de sa connaissance, soit un

3 A. Tarrius, "Territoires circulatoires des entrepreneurs commerciaux maghrébins de Marseille: du commerce communautaire aux réseaux de l'économie souterraine mondiale", Journal des anthropologues, n 59, 1995 , pp. 15-35. 
membre de sa parenté élargie, soit une personne originaire de la même vallée ou du même district que lui. Si la relation est plus distante, la présence d'un intermédiaire qui se porte garant auprès des deux parties est requise. Le hawâladâr transmet à ses parte- naires une lettre mentionnant les détails de la transaction (lettre b dans le schéma ci-dessous) et donne au migrant une autre lettre (lettre a) que celui-ci envoie à sa famille en Afghanistan par l'entremise d'un ami qui retourne chez les siens.

Schéma: Les transferts de fonds entre l'Iran, le Pakistan et l'Afghanistan dans les années 1990

IRAN

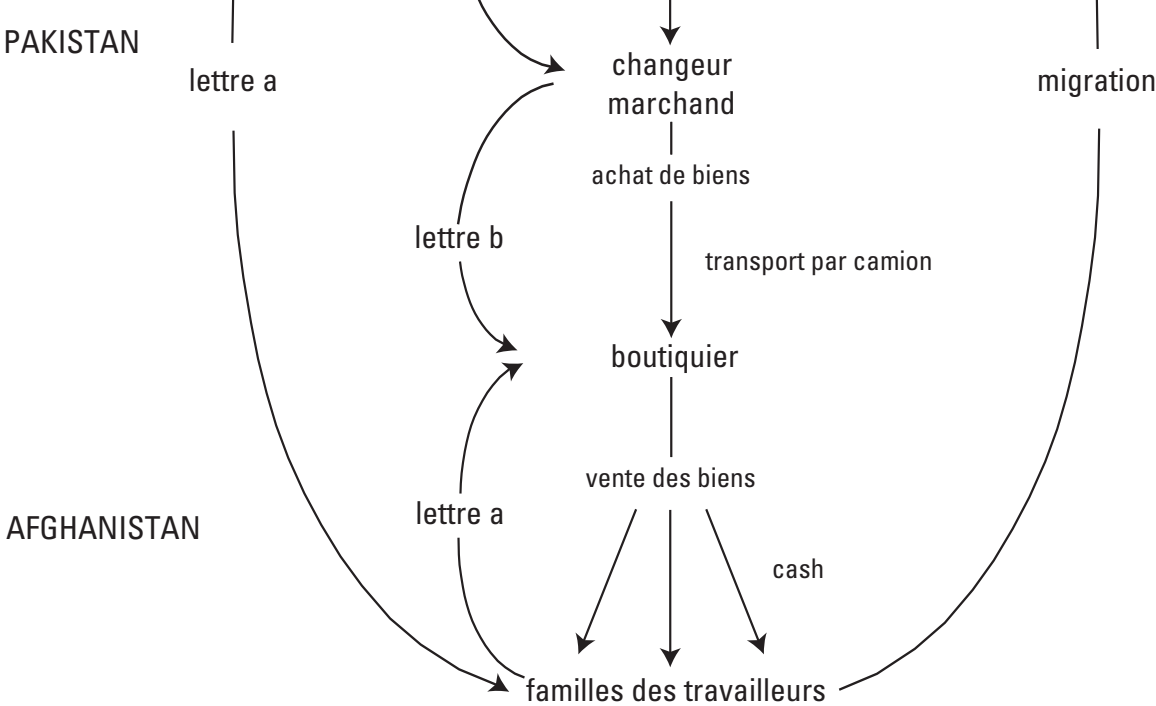

Source: A. Monsutti, Guerres et migrations: réseaux sociaux et stratégies économiques des Hazaras d'Afghanistan, Neuchâtel, Institut d'ethnologie; Paris, Maison des sciences de I'homme, 2004, p. 237. 
Après la chute du régime des talibans et I'instauration d'un gouvernement soutenu par la communauté internationale à Kaboul à la fin 2001, la plupart des transferts se font directement de I'Iran vers I'Afghanistan. Dans les années 1990 par contre, les tensions entre le grand pays chiite et les fondamentalistes sunnites rendaient tout franchissement de la frontière entre les deux pays particulièrement délicat. Le hawâladâr qui avait récolté des fonds en Iran les envoyait donc le plus souvent au Pakistan, tantôt par versement bancaire, tantôt sous forme de marchandises (en particulier des produits manufacturés: articles en plastique, habits ou tissus). Un partenaire y récupérait l'argent ou les biens qu'il revendait sur place avec un bénéfice. II pouvait utiliser les montants ainsi obtenus pour faire quelque profit supplémentaire (sur le marché du change par exemple), mais finissait par acheter d'autres marchandises (farine, riz, huile alimentaire, thé, sucre, mais aussi des marmites à pression ou des babouches en plastique). Cellesci étaient envoyées en camion jusqu'au Hazaradjat où un troisième partenaire tenait un magasin. Avec l'argent issu de la vente, le boutiquier remboursait les familles des migrants. La commission perçue pour cette transaction était basse ${ }^{4}$, car le profit du hawâladâr provenait essentiellement du fait qu'il faisait tourner ses affaires sans avoir forcément un important capital propre. En dépit du traumatisme de la guerre et de I'exil, les Hazaras ont ainsi été capables de tirer parti de la dispersion géographique et de la diversification économique qui en résultait en développant des structures transnationales de coopération particulièrement efficaces.

Comme en témoigne le présent volume, les débats concernant les effets des transferts de fonds dans les régions d'origine des migrants sont vifs. D'un côté, certains auteurs leur reprochent d'être souvent desti- nés à l'acquisition de biens de consommation et de prestige ou à des dépenses somptuaires, contribuant ainsi à l'accroissement des inégalités sociales. De l'autre, un nombre croissant d'agences de développement insistent au contraire sur le rôle potentiellement positif que ces envois d'argent peuvent jouer en apportant aux économies locales des capitaux qui peuvent ensuite être investis de façon productive. Dans le cas afghan, le système hawâla gagnerait sans aucun doute à être complété par un système bancaire qui permette de transformer le capital en fonds de crédit et d'investissement, de façon à ce qu'il soit plus utile à la réhabilitation des infrastructures du pays et qu'il participe à la création d'entreprises. Si l'émigration d'hommes jeunes peut poser à long terme des problèmes à la reconstruction de I'Afghanistan, il s'agit néanmoins d'une stratégie de survie qui a démontré son efficacité. Le système hawâla a permis à de nombreuses familles afghanes de se nourrir, alors que l'action humanitaire s'était surtout concentrée sur les réfugiés pendant l'occupation soviétique, puis s'était progressivement tarie pendant les années 1990 avant de s'amplifier à nouveau de façon désordonnée après la chute des talibans en 2001.

Mais au-delà des apports et des limites proprement économiques des envois d'argent effectués par les migrants, il faut insister sur leur dimension sociale. C'est par ce biais que des personnes éparpillées restent en contact et forment toujours un groupe d'entraide et de solidarité. II s'agit là d'un aspect essentiel des transferts de fonds: ils contribuent à produire et reproduire les relations sociales malgré la dispersion et - dans le contexte qui nous occupe ici - la guerre. C'est à ce niveau que se situe un de leurs rôles les plus cruciaux. En se penchant sur le système hawâla, entrelacs complexe de relations de coopération et de compétition, de confiance et de méfiance, on peut comprendre comment la dispersion géo-

4 En général, elle ne dépasse pas $3 \%$ entre I'Iran, le Pakistan et l'Afghanistan. Elle est plus élevée lorsque l'envoi est effectué à partir d'un pays occidental. 
graphique et la diversification économique qui en résulte permettent de répartir les risques et peuvent devenir une stratégie planifiée. En ce sens, le système hawâla est l'un des élé- ments qui expliquent que la société afghane n'a pas sombré dans un chaos hobbesien malgré les combats et le déplacement forcé de plusieurs millions de personnes.

\section{Références citées}

Colville, Rupert, "Afghan Refugees: Is International Support Draining Away after Two Decades in Exile?», Refuge, vol. 17, $n^{0} 4,1998$, pp. 6-11.

Hanifi, M. Jamil, "Anthropology and the Representations of Recent Migrations from Afghanistan», in E.M. Gozdziak and D.J. Shandy (eds.), Rethinking Refuge and Displacement: Selected Papers on Refugees and Immigrants, vol. 8, Arlington, American Anthropological Association, 2000, pp. 291-321.

Monsutti, Alessandro, Guerres et migrations: réseaux sociaux et stratégies économiques des Hazaras d'Afghanistan, Neuchâtel, Institut d'ethnologie; Paris, Maison des sciences de I'homme, 2004.

Tarrius, Alain, "Territoires circulatoires des entrepreneurs commerciaux maghrébins de Marseille: du commerce communautaire aux réseaux de l'économie souterraine mondiale», Journal des anthropologues, $\mathrm{n}^{0}$ 59, 1995, pp. 15-35. 